\title{
High performance radar images modelling and recognition of real objects
}

\author{
D A Zherdev ${ }^{1,2}$, V V Prokudin ${ }^{1}$
}

${ }^{1}$ Samara National Research University, Moskovskoe Shosse 34A, Samara, Russia, 443086

${ }^{2}$ Image Processing Systems Institute of RAS - Branch of the FSRC "Crystallography and Photonics" RAS, Molodogvardejskaya street 151, Samara, Russia, 443001

e-mail: t_treasure@mail.ru

\begin{abstract}
In the work there is a modernization of the parallel algorithm for the radar images formation of 3D models with the synthesis of the antenna aperture. In the formation of the scene description, the various structures are used in which it is possible to use more efficient and derived calculations. In addition, it is the topical task to recognize objects on radar images. Thus, on the basis of the implemented parallel program for modelling, the high performance required for simulating multiple radar images can be achieved.
\end{abstract}

\section{Introduction}

This research is a continuation of ideas and methods used in [1], where high-performance radar images modeling approach was considered. The goal of this work is to obtain a greater acceleration of the parallel program for synthetic aperture radar modeling by building a kd tree describing a threedimensional scene [2-4]. We used CUDA to perform a high-performance computing on a graphic card and achieve this goal. It is the main difficulty to form a trajectory signal along with the radar travel and then compute a radar image. In this study, an algorithm for obtaining radar characteristics was implemented with the construction of the kd tree structure, that allow to describe any threedimensional scene.

\section{Modelling and recognition}

The main computationally expensive part of the radar images modelling algorithm is in the processing of radiated and reflected radar signals. In this work, a modification of the CUDA algorithm was implemented, which difference at the stage of the trajectory signal constructing is in a previously calculated kd-tree for any three-dimensional scene. The subsequent execution of the synthesis of the radar aperture was performed the same way as discussed in [1]. This approach of computable operations reducing allowed us to achieve a three-fold acceleration compared with the previous algorithm implementation.

In addition, during the research, we carried out the experiments of object recognition in radar images. There are many methods and approaches of object recognition, among which the popular methods are convolutional neural networks [5], support vectors, nearest neighbours, etc. [6]. In this work, the object recognition was performed using the method of support subspaces [7]. We used three- 
dimensional models of the tank, BMP, BTR to construct the radar images. All sizes of the models were matched to the corresponding sizes of their real prototypes at the three-dimensional coordinate system. We used the modelling parameters presented in Table 1 when generated the training set. The bearing angle was $17^{\circ}$, which corresponds to the conditions of the real obtained SAR images training dataset. We modelled 100 images for each object. The step of rotation was equal the $3.6^{\circ}$ in the observation plane. Figure 3 shows the real and modeled SAR images of BTR.

There are the results of research shown below which related to the construction of a training model sample and the subsequent recognition of real images using model images at the training stage. Figure 1, a), b) shows the real radar images of a tank from the widely known MSTAR database, and figure 2, a), b) shows radar images obtained by modelling using the synthetic aperture radar method, with angles the bearing angle of $17^{\circ}$ and $15^{\circ}$ and the aspect angles are $17^{\circ}$ and $100^{\circ}$, respectively.

Table 1. Modelling parameters.

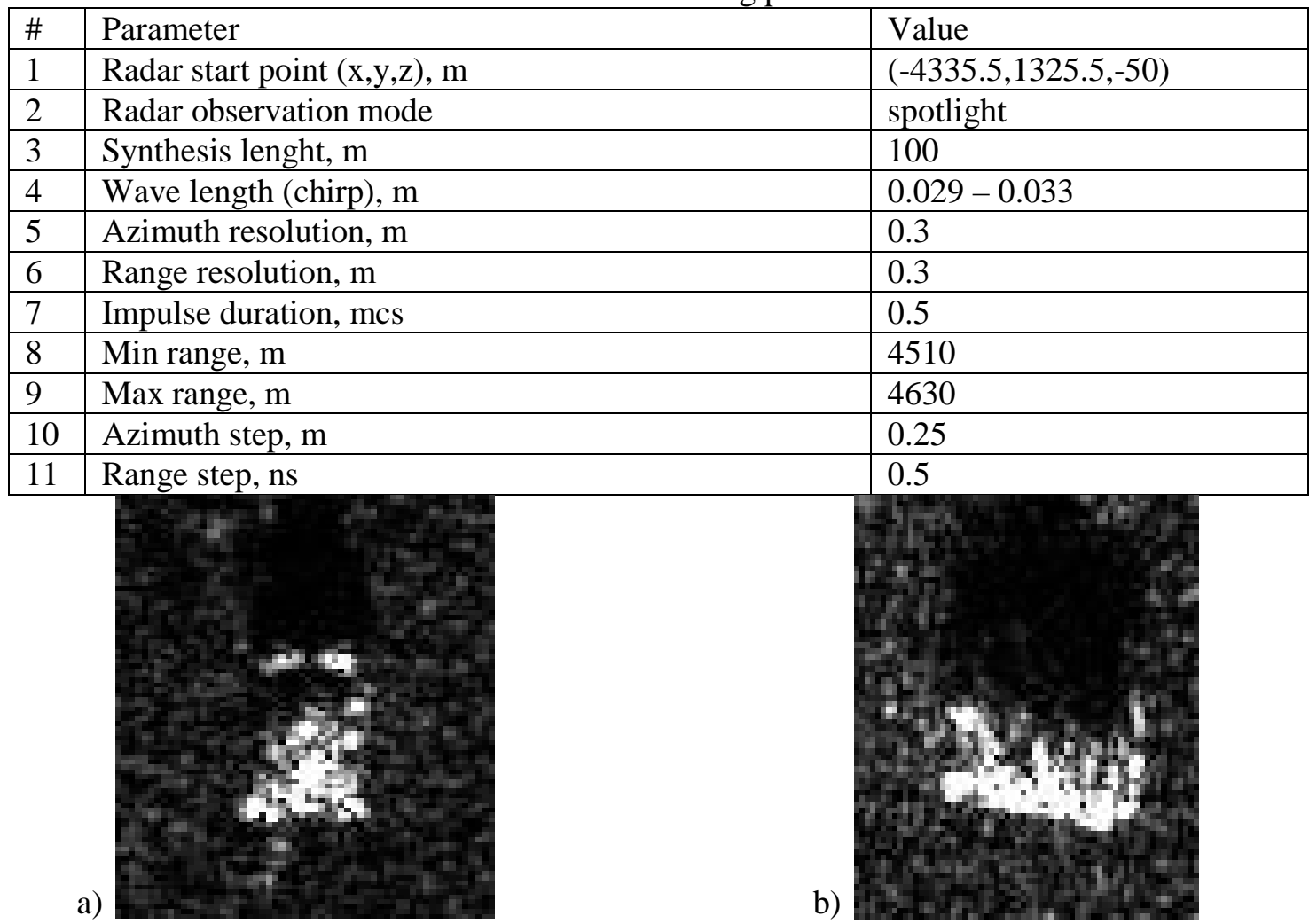

Figure 1. MSTAR dataset SAR images: a) bearing angle $17^{\circ}$, elevation angle $15^{\circ}$; b) bearing angle $17^{\circ}$, elevation angle $100^{\circ}$.

a)

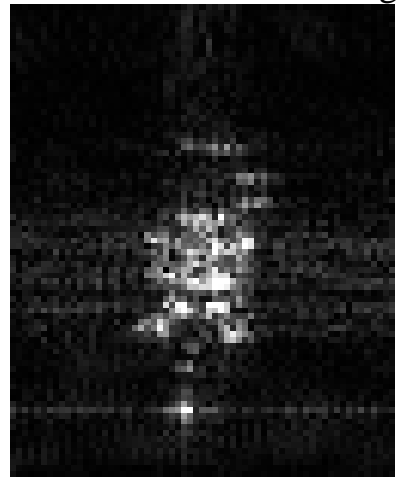

b)

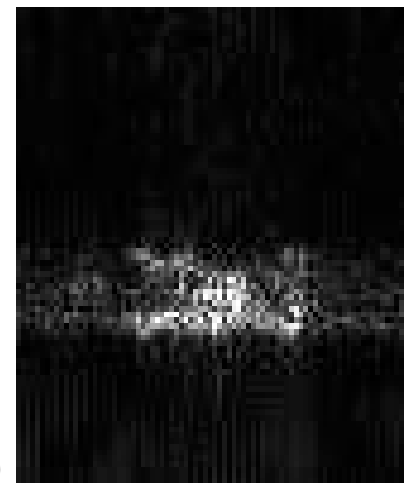

Figure 2. Modelled SAR images: a) bearing angle $17^{\circ}$, elevation angle $15^{\circ}$; b) bearing angle $17^{\circ}$, elevation angle $100^{\circ}$. 
The recognition results for three classes obtained using the recognition contingency index based algorithm [7] (without subclassing) are listed in Table 2. Note that the result of $62.78 \%$ correct recognition of the three classes was obtained on a relatively small training set (300 images). The MSTAR training sample contains 587 of image samples.

a)

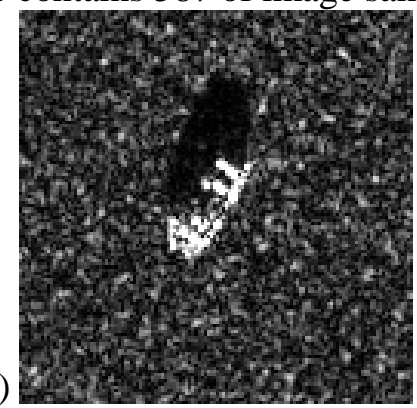

b)

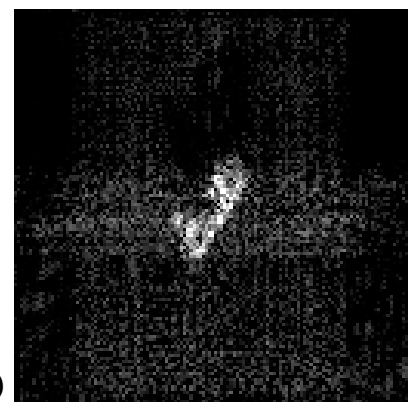

Figure 3. SAR images: a) model and b) real BTR target.

Table 2. Recognition results with modelled dataset training.

\begin{tabular}{|l|l|l|l|l|}
\hline \multicolumn{4}{|l|}{ Evaluated class } & \\
\hline & BMP2 & BTR70 & T72 & \\
\hline BMP2 & 402 & 124 & 61 & $68.48 \%$ \\
\hline BTR70 & 134 & 43 & 19 & $21.93 \%$ \\
\hline T72 & 119 & 51 & 412 & $70.79 \%$ \\
\hline
\end{tabular}

At the binary classification: the BMP and tank using the same algorithm, the result of $80.24 \%$ was achieved. The recognition results of the two classes without division into subclasses are shown in Table 3.

Table 3. Results of two objects recognition.

\begin{tabular}{|l|l|l|l|}
\hline \multicolumn{4}{|l|}{ Evaluated class } \\
\hline & BMP2 & T72 & \\
\hline BMP2 & 496 & 91 & $84.50 \%$ \\
\hline T72 & 140 & 442 & $75.94 \%$ \\
\hline
\end{tabular}

In addition, we carried out the experiment of the real images recognition by training on model images, which obtained using the ray tracing approach [8]. Table 4 shows the recognition results. The total percentage of correct recognition was $27.6 \%$. These results show that images modelled via raytracing is not quite suitable for recognition real images. Perhaps they can be used to create a simplified model of a three-dimensional scene.

Table 3. Results of objects recognition (dataset modelled via raytracing).

\begin{tabular}{|c|c|c|c|c|}
\hline & \multicolumn{3}{|c|}{ Evaluated class } & \\
\hline & BMP2 & BTR70 & T72 & \\
\hline BMP2 & 207 & 270 & 221 & $29.66 \%$ \\
\hline BTR70 & 78 & 67 & 51 & $34.18 \%$ \\
\hline T72 & 331 & 146 & 105 & $18.04 \%$ \\
\hline
\end{tabular}

It should be noted that in the MSTAR database, the training sample has 587 images. In contrast to presented model images dataset, the rotation of an object in the MSTAR images was performed with an irregular step and with rather large positioning errors.

\section{Conclusion}

The paper shows that the results of objects recognition using real images has the ability of the effective usage at the developed software. The images were obtained by modeling can form the training dataset at the proposed algorithm. In addition, the parallel algorithm acceleration is obtained 
by kd-tree construction let us help to perform high-computing and effective scattering calculation on the various object surfaces.

\section{References}

[1] Zherdev D A, Prokudin V V and Minaev E Y 2018 HPC implementation of radar images modelling method using CUDA Journal of Physics: Conference Series 1096012083

[2] Horn D R, Sugerman J, Houston M and Hanrahan P 2007 Interactive kd tree GPU raytracing Proceedings of the symposium on Interactive $3 D$ graphics and games 167-174

[3] Wehr D, Radkowski R 2018 Parallel kd-tree construction on the gpu with an adaptive split and sort strategy International Journal of Parallel Programming 46(6) 1139-1156

[4] Vinkler M, Havran V and Bittner J 2016 Performance Comparison of Bounding Volume Hierarchies and Kd-Trees for GPU Ray Tracing Computer Graphics Forum 35(8) 68-79

[5] Savchenko A V 2018 Trigonometric series in orthogonal expansions for density estimates of deep image features Computer Optics 42(1) 149-158 DOI: 10.18287/2412-6179-2018-42-1149-158

[6] Borodinov A A, Myasnikov V V 2018 Classification of radar images with different methods of image preprocessing CEUR Proceedings 2210 6-13

[7] Fursov V, Zherdev D and Kazanskiy N 2016 Support subspaces method for synthetic aperture radar automatic target recognition International Journal of Advanced Robotic Systems 13(5) DOI: $10.1177 / 1729881416664848$

[8] Zherdev D A, Fursov V A 2015 Support plane method applied to ground objects recognition using modelled SAR images Applications of Digital Image Processing XXXVIII International Society for Optics and Photonics 9599

\section{Acknowledgments}

The work was funded by the Russian Federation Ministry of Education and Science (agreement 007GZ/Ch3363/26) and RFBR (project \# 17-29-03112 ofi_m). 